\title{
O uso da mineração de dados como ferramenta para tomada de decisões em enfermagem
}

\author{
The use of data mining as a tool for decision making in nursing \\ El uso de minería de datos como una herramienta para la toma de decisiones en \\ enfermería
}

Rodrigo José Gomes ${ }^{1 *}$, Isadora Souza Silva², Chennyfer Dobbins Abi Rached³.

\begin{abstract}
RESUMO
Objetivo: Descrever as evidências científicas veiculadas na área da saúde sobre o uso da mineração de dados como ferramenta de processamento de dados e sua eficácia em auxiliar na tomada de decisão em enfermagem. Métodos: A metodologia utilizada foi a revisão integrativa. Para seleção das publicações foram utilizadas as plataformas online de bases de dados: Lilacs, Scielo, MedLine e os Periódicos da Capes. A busca das publicações foi realizada com os descritores: mineração de dados, tomada de decisões e enfermagem, seguindo a lógica booleana: and. Foram utilizados artigos entre os anos de 2000 e 2017. Resultados: $O$ estudo resultou em nove artigos, distribuídos nas áreas de Saúde Pública, Psiquiatria e Pesquisa, tendo como área das publicações Enfermagem (cinco) e Medicina (quatro). Percebe-se o envolvimento de 30 autores, dentre estes $26 \%(n=8)$ enfermeiros, $23 \%(n=7)$ médicos e $23 \%(n=7)$ da área do TI. Conclusão: Conclui-se ao finalizar esta pesquisa que a mineração de dados é sim uma ferramenta eficaz para o auxílio na tomada de decisões na área da enfermagem, uma vez que o método tradicional de análise de dados se tornou inviável pelo alto volume de dados armazenados.
\end{abstract}

Palavras-chave: Mineração de dados, Tomada de decisões, Enfermagem.

\begin{abstract}
Objective: To describe the scientific evidence in the area of health reports on the use of data mining as a tool for data processing and your effectiveness in assisting in decision-making in nursing. Methods: The methodology used was the integrative review. For selection of the publications have been used online platforms of databases: Lilacs, Scielo, MedLine and journals of Capes. The search of the publications was held with the descriptors: data mining, decision making and nursing, following the Boolean logic: and have been used articles between 2000 and 2017. Results: The study resulted in nine articles, distributed in the areas of Public Health, psychiatry and research, with the area of Nursing publications (five) and medicine (four). You can see the involvement of 30 authors, among them $26 \%(n=8)$ nurses, $23 \%(n=7)$ doctors and $23 \%(n=7)$ of the area you. Conclusion: It is concluded at the end of this research that data mining is an effective tool to aid decision-making in the field of nursing, since the traditional method of data analysis became impractical for high-volume data stored.
\end{abstract}

Key words: Data mining, Decision making, Nursing.

\footnotetext{
1 Universidade Estácio de Sá - UNESA. Rio de Janeiro - Brasil. ORCID: https://orcid.org/0000-0003-05002198 *E-mail: enfrodrigogomes@outlook.com

${ }^{2}$ Pontifícia Universidade Católica do Paraná - PUCPR. Curitiba - Brasil. ORCID: https://orcid.org/0000-00034666-3910

${ }^{3}$ Universidade Nove de Julho - UNINOVE. São Paulo - Brasil. ORCID: https://orcid.org/0000-0002-44993716
}

SUBMETIDO EM: 1/2019 


\section{RESUMEN}

Objetivo: Describir la evidencia científica en el área de la salud informes sobre el uso de minería de datos como herramienta para el procesamiento de datos y su eficacia para ayudar en la toma de decisiones en enfermería. Métodos: La metodología utilizada fue la revisión Integrativa. Para la selección de las publicaciones se han utilizado plataformas online de bases de datos: Lilacs, Scielo, MedLine y revistas de cabos. La búsqueda de las publicaciones se realizó con los descriptores: datos de explotación minera, de la decisión haciendo y cuidando, siguiendo la lógica booleana: y han sido los artículos usados entre 2000 y 2017. Resultados: El estudio resultó en nueve artículos, distribuidos en las áreas de salud pública, la psiquiatría y la investigación, con el área de enfermería publicaciones (cinco) y medicina (cuatro). Se puede ver la participación de 30 autores, entre ellos $26 \%(n=8)$ enfermeras, $23 \%(n=7)$ médicos y $23 \%(n=7)$ de la zona. Conclusión: Se concluye al final de esta investigación que la minería de datos es una herramienta eficaz para facilitar la toma de decisiones en el campo de la enfermería, puesto que el método tradicional de análisis de datos se convirtió en poco práctico para grandes volúmenes de datos almacenados.

Palabras clave: Minería de datos, Toma de decisiones, Enfermería.

\section{INTRODUÇÃO}

Atualmente o registro de dados realizado manualmente no âmbito hospitalar vem sendo deixado no passado sendo substituídos pelos sistemas informatizados que armazenam as informações em banco de dados que agilizam a procura, interpretação e a confiabilidade dos dados registrados. Já faz algum tempo que os hospitais e clínicas tanto privadas quanto públicas estão informatizando seus processos de armazenamento de dados para maior fidedignidade dos dados armazenados. Isto por sua vez facilita a busca por dados e a melhor tomada de decisão para a seleção de uma intervenção. Uma das técnicas que podem ser utilizadas para extração de informações relevantes para tomada de decisão rápida é a mineração de dados.

O uso da informação de maneira eficaz e eficiente se torna um elemento essencial para o sucesso das organizações, sendo até incorporado a seu patrimônio. Saber que a informação é um dos principais recursos estratégicos que a organização dispõe, requer que estas informações estejam estruturadas, disponíveis e sejam íntegras, condições que apenas se fazem possíveis com o uso de tecnologias computacionais, frequentemente conhecidas como Tecnologia da Informação e Comunicação, ou Sistemas de Informação (DALFOVO, 2007).

O Knowledge Discovery in Databases - KDD é constituído de três passos básicos que são o préprocessamento, a mineração propriamente dita e o pós-processamento ou interpretação dos resultados. Vemos então que o termo mais conhecido, Mineração de Dados, é na verdade uma das etapas do processo de descobrimento de conhecimento em bancos de dados. Em geral, a execução da tecnologia de KDD é dividida em 3 etapas principais: (I) Pré-Processamento, (II) Mineração de Dados e (III) Pós- Processamento.

O Pré-processamento dos dados é fundamental no processo de KDD, pois os dados coletados caracterizam a eficiência dos algoritmos de mineração aplicados. É nesta etapa que são realizadas as tarefas que eliminam os dados excessivos e conflitantes, recuperam-se os dados incompletos e se avaliam os possíveis dados discrepantes ao conjunto, chamados de outliers (DALFOVO, 2007).

A Transformação de Dados é a fase do KDD que antecede a fase de Data Mining. Após serem escolhidos, limpos e pré-processados, os dados devem ser armazenados e formatados de maneira correta para que os algoritmos possam ser aplicados. Todas as etapas do processo são importantes para o sucesso do mesmo. No entanto, é a etapa de Mineração de Dados (Data Mining - DM) que recebe o maior destaque na literatura (PRASS, 2012).

Conforme BERRY e LINOFF (1997), Data Mining nada mais é que a exploração e apreciação, de forma automatizada de grandes bases de dados para descobrir padrões e regras. A mineração de dados objetiva 
fornecer as informações que permitam as corporações, montar melhores planos de ação, intervenções e suporte, visando a melhoria do processo ou negócio. Trata-se da principal etapa do processo de KDD. É aqui que efetivamente o conhecimento é buscado a partir dos dados aplicando-se algoritmos. Estes algoritmos procuram explorar só dados de forma a produzir conhecimento, baseados em determinados paradigmas. Temos alguns exemplos de algoritmos que podem ser aplicados e o que podemos encontrar com a aplicação de cada um:

a) Classificação - arruma os dados em grupos predefinidos; b) Clustering - arruma os dados em grupos não predefinidos encontrando padrões de semelhança entre os dados; c) Regressão - tenta encontrar uma função que modele os dados com o menor erro possível; e, d) Aprendizado de regras de associação - procura por relacionamentos entre as variáveis (BATISTA AC, 2009).

De acordo com PRASS (2012), a Mineração de Dados traz uma série de conceitos e metodologias para uma ampla variedade de campos. Estatísticos, pesquisadores de Inteligência Artificial (IA) e administradores de bancos de dados usam técnicas diferentes para interpretar e avaliar os resultados obtidos com a mineração para, no final, chegar a um mesmo fim: a informação. A mineração de Dados na área da saúde necessita de um especialista para realizar a interpretação e avaliação dos dados obtidos, sendo estes enfermeiros, médicos, fisioterapeutas, farmacêuticos, biomédicos e demais profissionais especialistas em suas determinadas áreas de atuação.

Atualmente a informação e o conhecimento são vantagens legais, estratégicas e indispensáveis à busca de maior autonomia nas ações das empresas de saúde, controle social e na tomada de decisão com prazos cada vez mais curtos. Na saúde, especialmente no setor público, o emprego desta técnica está sendo aceito como uma forma de aprimorar a busca de conhecimento. Além disso, o emprego do KDD nas grandes bases de dados de saúde colabora para a descoberta de relações integradas para que possa ser feita uma previsão de disposições futuras baseadas nos acontecimentos ocorridos. Permite a diferenciação de pacientes que buscam assistência, identifica terapias médicas de sucesso para diferentes morbidades e evidencia novos padrões de agravos. (DANTAS, et al. 2008)

Diante deste cenário, emergiu a necessidade de se conhecer o uso da mineração de dados na área da saúde, sobretudo da enfermagem a partir da pergunta norteadora: quais as evidências científicas veiculadas na área da saúde/enfermagem sobre o uso da mineração de dados como ferramenta do processamento de dados? Portanto, o presente estudo teve como objetivo descrever as evidências científicas veiculadas na área da saúde sobre o uso da mineração de dados como ferramenta de processamento de dados.

\section{MÉTODOS}

Para atingir o objetivo proposto, utilizou-se como metodologia de pesquisa a revisão integrativa. A busca das publicações foi realizado com os Descritores em Ciências da Saúde (DeCS) e com o Medical Subject Headings (MeSH) combinados da seguinte forma: mineração de dados, tomada de decisões e enfermagem, seguindo a lógica booleana: and. Para seleção das publicações foram utilizadas as plataformas online de bases de dados: Lilacs, Scielo, MedLine e os Periódicos da Capes.

Durante a busca nas bases de dados foram utilizados como critério de inclusão artigos nacionais e internacionais, a disponibilidade da publicação na íntegra, nos idiomas inglês, espanhol ou português, possuir um ou mais dos descritores utilizados para a busca nas bases de dados, serem de acesso livre/gratuito e estar estruturado em formato de artigo. Os critérios para exclusão foram: produções duplicadas; artigos pagos; artigos de revisões de literatura, dissertações ou teses e publicações que não atendiam ao objeto do estudo ou que não respondiam à pergunta norteadora.

Após a identificação dos artigos os pesquisadores leram todos os resumos separadamente, para identificar os que atendiam aos critérios de inclusão. Foi executada uma busca de forma mais ampla, sendo utilizada as bases de dados, pesquisa em periódicos e quando necessário contatando autores. 
Foram identificados 22 artigos publicados que abordavam o tema de mineração de dados aplicado à área da saúde; sendo 7 na Lilacs, 2 na Scielo, 6 na MedLine e 7 na Capes Periódicos. Após leitura dos resumos realizada pelos pesquisadores e aplicação dos critérios de inclusão, foram excluídos 13 artigos da pesquisa, destes 1 era artigo de revisão e 12 artigos que não possuíam relação com a pergunta norteadora ou com o tema. Restando 9 artigos que compuseram a amostra, conforme apresentado na figura 1.

Figura 1 - Distribuição dos artigos encontrados, excluídos e selecionados, segundo os meios eletrônicos. Curitiba, Brasil, 2019.

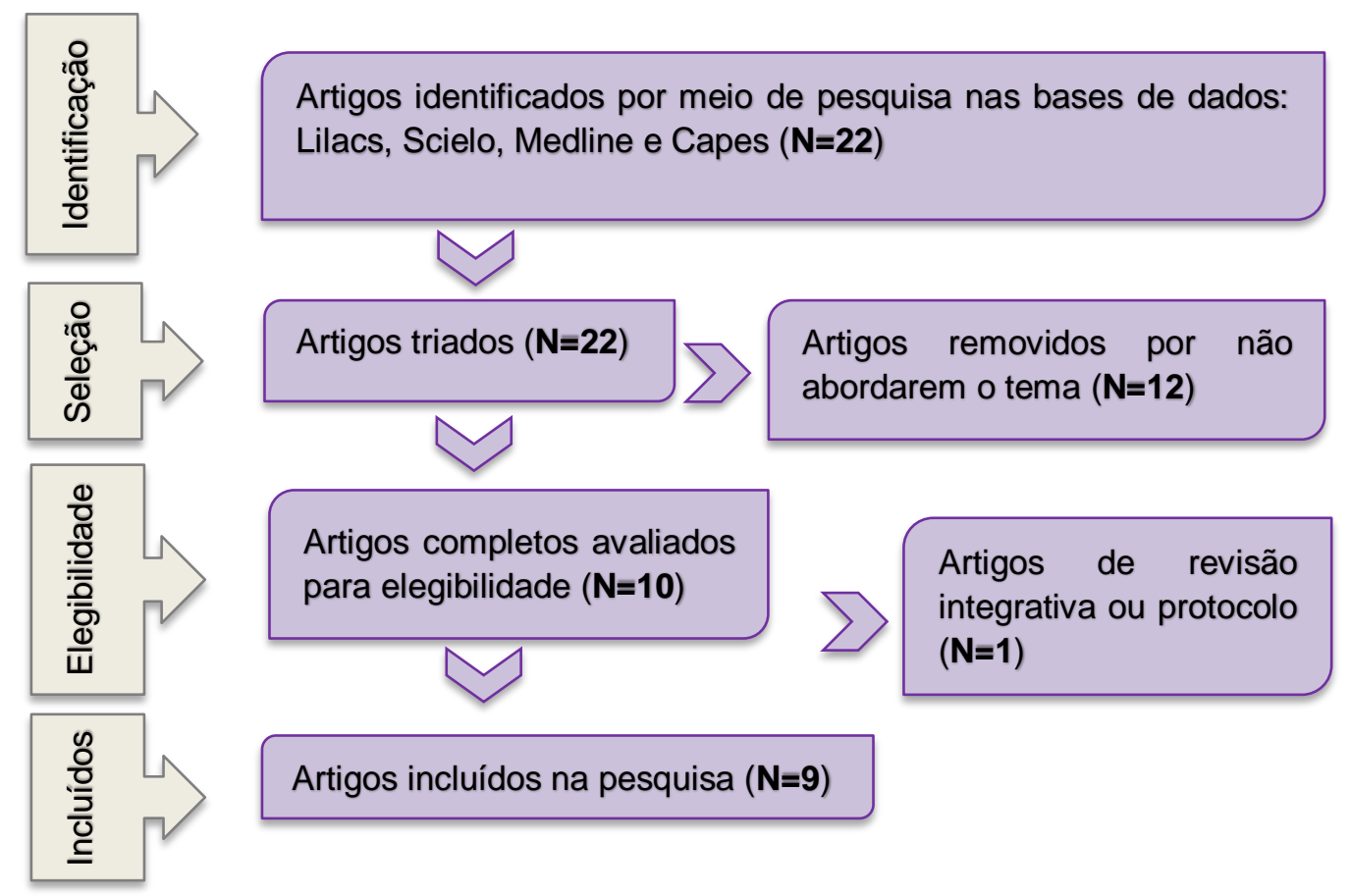

Fonte: Dados de pesquisa, 2019.

\section{RESULTADOS E DISCUSSÃO}

A pesquisa resultou em nove artigos, distribuídos nas áreas de Saúde Pública, Psiquiatria e Pesquisa, tendo como área das publicações Enfermagem $(n=5)$ e Medicina $(n=4)$. Percebe-se o envolvimento total de 30 autores, dentre estes 26\% ( $n=8)$ enfermeiros, 23\% ( $n=7)$ médicos e 23\% ( $n=7)$ da área do TI. Destaca-se grande envolvimento de profissionais da área de TI no tema pesquisado.

Estas publicações envolvem em sua totalidade 9 revistas, que foram categorizadas de acordo com 0 Qualis: $11 \%(n=1)$ da categoria $A, 66 \%(n=6)$ da categoria B, $11 \%(n=1)$ da categoria $C$ e $11 \%(n=1)$ não categorizadas. Com relação à indexação dos nove artigos, destacou-se o banco de dados da Lilacs com cinco publicações, o portal da Capes com duas publicações, Scielo com uma publicação e um artigo na base de dados MedLine. Os artigos selecionados foram organizados em Quadros para melhor visualização das variáveis de análise.

Observa-se que $33 \%(n=3)$ dos artigos foram publicados no ano de 2017 e $33 \%(n=3)$ no ano de 2016; $22 \%(n=2)$ em 2015 e 11\% ( $n=1)$ em 2005. Verificou-se que entre a primeira publicação em 2005 e a posterior em 2015, houve um lapso temporal de 10 anos; porém, se vê o contrário entre 2015 e 2017, com aumento do crescimento das publicações. Isso deve-se talvez ao atual crescimento e utilização de bases de dados no âmbito hospitalar, pois, como dito anteriormente a mineração de dados auxilia na tomada de decisões clínicas. O Brasil é o país que mais publicou sobre o tema nos últimos anos com $55 \%(n=5)$ do total, Baltimore $11 \%$ 
$(n=1)$, Cuba 11\% $(n=1)$, Colômbia 11\% $(n=1)$ e Austrália 11\% $(n=1)$ dos artigos analisados. No Quadro 1 considerou-se: autoria, algoritmo utilizado, periódico, Qualis, ano, país e a base de dados.

Quadro 1 - Publicações cientificas segundo autor, algoritmo utilizado, periódico, Qualis, ano, país e a base de dados. Curitiba, Brasil, 2019.

\begin{tabular}{|c|c|c|c|c|c|c|c|}
\hline Ordem & Autoria & $\begin{array}{l}\text { Algoritmo } \\
\text { Utilizado }\end{array}$ & Periódico & Qualis & Ano & País & Base \\
\hline A & $\begin{array}{l}\text { SARTORELLI AP, } \\
\text { CUBAS MR. }\end{array}$ & J48 | NPP & $\begin{array}{l}\text { Saúde \& Pesquisa, } \\
\text { Maringá }\end{array}$ & B3 & 2017 & Brasil & Lilacs \\
\hline B & $\begin{array}{l}\text { BATTERHAM M, } \\
\text { et al. }\end{array}$ & CART | AUC & $\begin{array}{l}\text { Nutrition \& dietetics: } \\
\text { journal of the dietitian's } \\
\text { association of Australia. }\end{array}$ & B2 & 2017 & Austrália & Lilacs \\
\hline C & FOJO AT, et al. & CART & $\begin{array}{l}\text { Journal of Psychiatric } \\
\text { Research }\end{array}$ & A1 & 2017 & Baltimore & MedLine \\
\hline D & $\begin{array}{ll}\text { MOREIRA } & \text { LB, } \\
\text { NAMEN AA. }\end{array}$ & C45 | CART & $\begin{array}{l}\text { Journal of Health } \\
\text { Informatics }\end{array}$ & B3 & 2016 & Brasil & Lilacs \\
\hline E & TORRES JA, et al. & C45 & $\begin{array}{l}\text { Revista Cubana de } \\
\text { Pediatria }\end{array}$ & * & 2016 & Cuba & Lilacs \\
\hline $\mathbf{F}$ & $\begin{array}{l}\text { MEDEIROS LB et } \\
\text { al. }\end{array}$ & C4.5 | Weka & Ciência \& Saúde Coletiva & B1 & 2016 & Brasil & Scielo \\
\hline G & $\begin{array}{l}\text { GALLEGO JA, et } \\
\text { al. }\end{array}$ & k-Means & Biosalud & C & 2015 & Colômbia & Lilacs \\
\hline H & $\begin{array}{l}\text { FRANÇA G, } \\
\text { CARVALHO D. }\end{array}$ & J48 | Weka & $\begin{array}{l}\text { Revista de Gestão em } \\
\text { Sistemas de Saúde }\end{array}$ & B4 & 2015 & Brasil & Capes \\
\hline I & $\begin{array}{l}\text { STEINER MTAM, } \\
\text { et al. }\end{array}$ & C4.5 & Gestão \& Produção & B1 & 2005 & Brasil & Scielo \\
\hline
\end{tabular}

Fonte: Dados de pesquisa, 2019.

Quadro 2 - Publicações cientificas segundo objetivo geral da pesquisa. Curitiba, Brasil, 2019,

\begin{tabular}{|c|l|}
\hline Ordem & \multicolumn{1}{c|}{ Objetivo geral } \\
\hline A & Complementar os fatores relacionados a mortalidade infantil. \\
\hline B & $\begin{array}{l}\text { O objetivo desta análise foi demonstrar e comparar a utilidade dos métodos de mineração de dados na } \\
\text { classificação de um resultado categórico derivado de uma intervenção relacionada à nutrição. }\end{array}$ \\
\hline C & $\begin{array}{l}\text { Para doenças psiquiátricas, existe informação rica na medição em série de sintoma de saúde mental } 22 \\
\text { pontuações. }\end{array}$ \\
\hline D & $\begin{array}{l}\text { Descrever uma aplicação que, utilizando-se de técnicas de mineração de dados, visa auxiliar os } \\
\text { especialistas no processo de diagnóstico de pacientes com suspeita clínica de Alzheimer. }\end{array}$ \\
\hline E & $\begin{array}{l}\text { Para detectar sintomas e sinais com valor preditivo para a dengue em pacientes pediátricos febris através } \\
\text { da técnica da Árvore de Decisão, a fim de aumentar a eficácia clínica do diagnóstico de tal doença. }\end{array}$ \\
\hline F & $\begin{array}{l}\text { Construir um modelo de suporte à decisão para auxiliar profissionais do serviço de saúde especializado a } \\
\text { identificar os padrões de comportamento no uso dos serviços da Estratégia de Saúde da Família das } \\
\text { pessoas vivendo com HIV/Aids atendidas no ambulatório. }\end{array}$ \\
\hline G & $\begin{array}{l}\text { Determine as relações entre fatores de risco nas mães mulheres grávidas, através de técnicas de mineração } \\
\text { de dados. }\end{array}$ \\
\hline H & $\begin{array}{l}\text { Esta pesquisa surgiu da necessidade de conhecer os padrões de relacionamento entre os diversos setores } \\
\text { do hospital com a área de informática, no que se refere à solicitação de atendimentos a problemas de } \\
\text { Tecnologia de Informação (TI). }\end{array}$ \\
\hline I & $\begin{array}{l}\text { Objetiva mostrar a influência da análise exploratória dos dados no desempenho das técnicas de Mineração } \\
\text { de Dados (Data Mining) quanto à classificação de novos padrões por meio da sua aplicação a um problema } \\
\text { médico, além de comparar o desempenho delas entre si, visando obter a técnica com o maior percentual de } \\
\text { acertos. }\end{array}$ \\
\hline
\end{tabular}

Fonte: Dados de pesquisa, 2019. 
Sobre o tema dos artigos destacaram-se sete $(77 \%)$ artigos que utilizaram a mineração de dados como ferramenta para implementar novos protocolos para melhoria de processos já existentes; e dois (22\%) utilizaram a mineração de dados como fonte de dados retroativos de tratamentos já utilizados e como ferramenta para tomada de decisões clínicas sobre qual o tratamento a ser utilizado futuramente. Referente a metodologia utilizada nos estudos analisados, demonstrou-se, na amostra: dois ensaios clínicos randomizados, cinco de estudo coorte retrospectivo, dois quantitativos descritivos. No Quadro 2 apresentamse o objetivo geral de cada pesquisa.

Quadro 3 - Publicações cientificas segundo resultados da pesquisa. Curitiba, Brasil, 2019.

\begin{tabular}{|c|c|}
\hline Ordem & Resultados Principais \\
\hline A & $\begin{array}{l}\text { Reforça a necessidade de políticas públicas voltadas à saúde materna, a evitabilidade de partos prematuros } \\
\text { e a maior qualidade de atenção aos nascidos prematuros. }\end{array}$ \\
\hline B & $\begin{array}{l}\text { Os métodos de mineração de dados podem fornecer uma alternativa para análise de dados exploratórios. } \\
\text { É necessária cautela com o método de mineração de dados utilizado. }\end{array}$ \\
\hline C & $\begin{array}{l}\text { A abordagem apresentada ilustra que os métodos analíticos inovadores para os dados existentes podem } \\
\text { ampliar seu alcance mais amplamente. O uso sistemático de medidas repetidas de sintomas psiquiátricos } \\
\text { oferece a promessa de medicina de precisão no campo da saúde mental. }\end{array}$ \\
\hline D & $\begin{array}{l}\text { A inclusão de outras hipóteses diagnósticas; a descoberta de novas características descritas no prontuário } \\
\text { médico, de forma a melhorar o poder preditivo dos modelos; a melhoria e ampliação das informações na } \\
\text { base de dados; e a utilização de método de grupos de modo a aumentar a acurácia dos classificadores } \\
\text { base. }\end{array}$ \\
\hline $\mathbf{E}$ & $\begin{array}{l}\text { Propõe regras de decisão com alta sensibilidade e valor preditivo, e conclui que as variáveis laboratoriais } \\
\text { e também levando em conta a contagem de plaquetas. }\end{array}$ \\
\hline $\mathbf{F}$ & $\begin{array}{l}\text { As decisões subsidiadas pelo modelo cooperam para a melhoria da assistência prestada às PVHA, através } \\
\text { de uma gestão do cuidado mais integrada e participativa, alcançando resultados satisfatórios que atendem } \\
\text { ao objetivo para o qual o modelo foi criado, confirmando sua eficácia. }\end{array}$ \\
\hline $\mathbf{G}$ & $\begin{array}{l}\text { Toda essa informação serve para fazer uma chamada as instituições que prestam saúde para concentrar } \\
\text { mais esforços em melhorar atenção às mulheres de tal forma a promover aconselhamento familiar, } \\
\text { programas de vacinação, cuidados Odontologia, participação em programas educação e promoção de } \\
\text { amamentação, a fim de assegurar que o Cuidado Primário em Saúde dê frutos positivos no menor tempo } \\
\text { possível. }\end{array}$ \\
\hline $\mathbf{H}$ & $\begin{array}{l}\text { Pode auxiliar tanto o gestor de TI como o do hospital na compreensão da qualidade de serviços } \\
\text { disponibilizados pelo setor a toda a instituição, indicando a necessidade de implementação, ou ajustes, das } \\
\text { ações de melhores práticas na TI. Da mesma forma, permite identificar quais são os problemas mais } \\
\text { relevantes vivenciados e direcionar melhor os esforços e investimentos de informática na instituição } \\
\text { hospitalar. A extração de conhecimento de bases bem estruturadas dá melhores condições de tomada de } \\
\text { decisão aos seus gestores e maior qualidade de governança do setor. }\end{array}$ \\
\hline $\mathbf{I}$ & $\begin{array}{l}\text { Os métodos apresentados podem ser utilizados nos mais diversos problemas reais de classificação. Deste } \\
\text { modo, especialistas das mais diversas áreas poderiam avaliar os resultados fornecidos pelos métodos } \\
\text { abordados e validar (ou não) a plausibilidade das predições realizadas por eles, tendo-se, pois, uma } \\
\text { ferramenta auxiliar para as suas tomadas de decisão. De posse do método validado com menor percentual } \\
\text { de erros, novos padrões poderiam ser classificados por ele, fornecendo um maior respaldo às tomadas de } \\
\text { decisão dos especialistas. }\end{array}$ \\
\hline
\end{tabular}

Fonte: Dados de pesquisa, 2019.

O pressuposto inicial de que a mineração de dados é realmente eficaz na tomada de decisão é bastante válido, uma vez que as informações obtidas a partir deste estudo comprovam sua eficácia e confiabilidade na pesquisa científica e aplicação clínica. No Quadro 3 são informados os resultados principais. Como constatação a eficácia da utilização da mineração de dados podemos analisar os resultados obtidos no quadro 3, que demonstra a conclusão de cada um dos artigos avaliados neste estudo.

Percebe-se que todos os artigos incluídos neste estudo reconhecem a mineração de dados como sistema de predição eficaz no auxílio a tomada de decisões. Porém, deve-se levar em consideração que existem algumas variáveis que devem ser observadas, como a utilização do algoritmo correto para análise dos dados, a confiabilidade dos dados inseridos na base de dados e a correta avaliação dos dados por especialista no assunto em questão. 
O processo de mineração de dados pode trazer uma estimada recompensa à área da saúde pois, com ela podemos identificar padrões de novas doenças e ter auxílio no direcionamento a uma rápida tomada de decisão. Observa-se que a KDD e a DM são ferramentas utilizadas na área de saúde e estão em processo de atualização e parametrização, entretanto, cabe ressaltar que essas ferramentas são apoio e não devem substituir a busca da informação prévia do domínio exploração (GALVAO, MARIN, 2009)

Este trabalho buscou apresentar informações que possam servir de reflexão para o setor saúde em relação ao processo de utilização da mineração de dados na busca do saber de bases de dados da área de ciências da saúde, caracterizando essas informações e entendimento como ferramenta de apoio na tomada de decisão das ações de saúde. Como limitações constatou-se a escassez de artigos do tema relacionado à enfermagem e área da saúde em geral. Deste modo sugerimos futuros estudos para complementação de lacunas no conhecimento e também agregação a produção científica na área da enfermagem sobre o tema proposto.

\section{CONSIDERAÇÕES FINAIS}

A Mineração de Dados atualmente caminha para uma popularização. As ferramentas, cada vez mais amigáveis e fáceis de serem usadas por usuários que não sejam especialistas em mineração de dados, desempenham um papel fundamental nesse sentido. O resultado da pesquisa sugere que a mineração de dados é sim uma ferramenta eficaz para o auxílio na tomada de decisões na área da enfermagem e na área da saúde em geral, uma vez que o método tradicional de análise de dados se tornou inviável pelo alto volume de dados armazenados. A mineração de dados realiza esta análise de forma mais rápida e eficaz, selecionando apenas as informações mais relevantes e necessárias. Não resta dúvida de que essa é uma área extremamente promissora e que, apesar dos resultados já obtidos, ainda tem muito para oferecer.

\section{REFERÊNCIAS}

1. BATISTA AC. Análise da aplicação de algoritmos de data mining em bases de dados de vendas de produtos. Universidade de Pernambuco. 2009.

2. BATTERHAM M, et al. Data mining: Potential applications in research on nutrition and health. Nutr Diet. 2017; 74(1):3-10.

3. CAMILO C, SILVA J. Mineração de Dados: Conceitos, tarefas, métodos e ferramentas. Univ Fed Goiás. 2009.

4. DALFOVO O. Modelo de integração de um sistema de inteligência competitiva com um sistema de gestão da informação e de conhecimento. UFSC; 2007.

5. DANTAS ERG, et al. O uso da descoberta de conhecimento em base de dados para apoiar a tomada de decisões. $V$ Simpósio Excel em Gestão e Tecnol. 2008; 1-10.

6. FOJO AT, et al. A precision medicine approach for psychiatric disease based on repeated symptom scores. J Psychiatr Res. 2017; 95:147-55.

7. FRANÇA G, CARVALHO D. Descoberta de padrões em ordens de serviço de tecnologia da informação em hospital. Rev Gestão em. 2016; 5:41-51.

8. GALLEGO JA, et al. Aplicación de técnicas de minería de datos en atención primaria en salud (APS) para el análisis de riesgos en mujeres gestantes de la población manizaleña atendida por Assbasalud. Biosalud. 2015; 14(2):71-8.

9. GANONG LH. Revisão integrativa na pesquisa de enfermagem. Adv Nurs Sci. 1987; 10(1).

10. GORDON S, et al. Data Mining Techniques: For Marketing, Sales, and Customer Support. Vol. 25, New York: Wiley Computer Publishing. 1997; 545p.

11. MEDEIROS LB, et al. Integração entre serviços de saúde no cuidado às pessoas vivendo com aids: uma abordagem utilizando árvore de decisão. Cien Saude Colet. 2016; 21(2):543-52.

12. MENDES KDS, et al. Revisão integrativa: método de pesquisa para a incorporação de evidências na saúde e na enfermagem. Texto Context - Enferm. 2008; 17(4):758-64.

13. MOREIRA LB, NAMEN AA. System predictive for Alzheimer is disease in clinical trial. J Heal Informatics. $2016 ; 8(3): 87-94$.

14. NEVES RCD. Pré-processamento no processo de descoberta de conhecimento em banco de dados. 2003.

15. PASTA A. Aplicação da técnica de data mining na base de dados do ambiente de gestão educacional: um estudo de caso de uma instituição de ensino superior de Blumenau-Sc. UNIVALI. 2011.

16. PRASS FS. Kdd - uma visão geral do processo. Texto \& Contexto UFSC. 2012.

17. SARTORELLI AP, CUBAS MR. Factors that contribute to children's mortality assessed through data mining. Saúde e Pesqui. 2017; 10(1):33-41.

18. STEINER MTAM, et al. Abordagem de um problema médico por meio do processo de KDD com ênfase à análise exploratória dos dados. Gestão \& Produção. 2006; 13:325-37.

19. TORRES JA, et al. Decision tree technique applied to the clinical method in the dengue diagnosis. Rev Cubana Pediatr. 2016; 88(4):441-53.

20. GALVAO ND, MARIN, HF. Técnica de mineração de dados: uma revisão da literatura. Acta Paul. Enferm., São Paulo, v. 22, n. 5, p. 686-690, Oct. 2009. 